\title{
Investigation of Flying-Height Stability of Thermal Fly-Height Control Sliders in Lubricant or Solid Contact with Roughness
}

\author{
Jinglin Zheng • David B. Bogy
}

Received: 28 October 2009/Accepted: 6 April 2010/Published online: 17 April 2010

(C) The Author(s) 2010. This article is published with open access at Springerlink.com

\begin{abstract}
When the magnetic spacing in hard disk drives is reduced to sub-3 $\mathrm{nm}$, contact between the slider and disk becomes inevitable. Stability analysis is used in this study to investigate the head-disk interface (HDI) stability of thermal fly-height control (TFC) sliders in light contact with the disk lubricant or solid roughness. We implement an improved DMT model with sub-boundary lubrication into the CML air bearing program and analyze the stability of equilibrium states of a TFC slider under different thermal actuations. It is found that stability is lost when the slider penetrates deeper into the lubricant layer, due to a fast growth in the adhesion force, and it is restored when the solid roughness contact develops. In addition, the critical point for the onset of this instability and the range of this instability region is found to vary with lubricant thickness and protrusion surface steepness, while keeping the air bearing design the same.
\end{abstract}

Keywords Magnetic recording ·

Thermal flying-height control · Head-disk interface . Interfacial forces · Instability

\section{Introduction}

To push the areal density in magnetic recording hard disk drives to 5-10 terabits per square inch, the magnetic spacing between the slider and the disk should be reduced to sub-3 nm. Excluding the thickness of the diamond-like-

\section{J. Zheng $(\bowtie) \cdot$ D. B. Bogy}

Computer Mechanics Laboratory, Department of Mechanical

Engineering, University of California, Berkeley, CA 94720,

USA

e-mail: zhengjinglin0420@gmail.com carbon (DLC) overcoats on both the slider and disk $(\sim 1 \mathrm{~nm})$, the surface roughness $(\sim 0.75 \mathrm{~nm})$, and the lubricant thickness $(\sim 1 \mathrm{~nm})$, the possible physical spacing or flying clearance is reduced to only $\sim 0.25 \mathrm{~nm}$. At such a small physical spacing, the slider's contact with the disk or at least with the lubricant layer becomes inevitable.

One promising scheme for such an ultra-high density recording is to have a part of the slider at the read/write transducer area in contact with the lubricant layer while most of the slider remains well above the disk, being supported by the air bearing. Thermal flying-height control (TFC) technology can be applied to produce a protruded part on the air bearing surface [1-3]. When actuated, this protruded part can penetrate into the lubricant layer during reading or writing. This regime was proposed in [4] as "surf-recording," and it potentially has the advantage of reducing the physical spacing while avoiding solid sliderdisk contact. A major concern associated with this approach is that the head-disk-interface (HDI) stability might be compromised by the increasingly significant interfacial forces (adhesion force, contact force, etc.) at the near-contact region as revealed in [5-7]. Experimentally, it is also found that the TFC slider often exhibits unstable bouncing vibrations when the actuation power is increased beyond a critical point (usually termed the "touch-down power") $[8,9]$. Thus, it is important to determine in what range the slider can sustain a stable flying-state to make the surf-recording scheme feasible. The investigations in $[4$, 10] used dynamic simulations to show that a lube-surfing status could be reached within certain protrusion height limits. In $[4,10]$, the lubricant was modeled as a soft solid layer that is plastically deformed under contact. A power law was adopted for calculating the contact force. For the adhesion force, the deformation profile at contact was not considered in $[4,10]$. In this study, we apply a stability 
analysis to a TFC slider in contact with a disk lubricant and possibly also roughness. By adopting a sub-boundary lubrication model [11] based on the classical DeryaginMuller-Toporov model (the DMT model), we include the effects of lubricant- and solid-contacting adhesion where the deformed contact profile is taken into account. The real TFC protrusion profile is applied for an industry-produced femto-sized slider $\left(0.85 \times 0.70 \times 0.23 \mathrm{~mm}^{3}\right)$. The equilibrium solution is examined as a function of the thermal protrusion height. An instability region is found as the slider comes into contact with the lubricant layer or the solid disk roughness. The effects of lubricant thickness and protrusion profile on the range of the instability region are also investigated.

\section{Adhesion and Contact Models}

To consider the effect of contact with the lubricant/disk roughness, asperity-based adhesion models are needed. In addition, the presence of a monolayer of lubricant must be considered as it makes a large contribution to the interfacial adhesion at extremely small head-disk spacing. Here, we adopt the sub-boundary lubrication model proposed by Stanley et al., which is based primarily on the improved DMT model. As both the slider and the disk surfaces are covered with DLC overcoats, the DMT model is more suitable for modeling the contact between these two solid surfaces. Furthermore, the existence of a lubricant layer and its thickness are also taken into account in this model. The problem of two contacting rough surfaces is first converted to the problem of the contact between a smooth plane and a nominally flat plane. Then the total adhesion force $F_{\mathrm{s}}$ between the two surfaces with sub-boundary lubrication is given by [11]:

$$
\begin{aligned}
F_{\mathrm{s}}= & \eta A_{\mathrm{n}}\left(\int_{-\infty}^{d-t} \frac{8}{3} \pi R \delta \gamma\left[\left(\frac{\varepsilon}{d-u-t+\varepsilon}\right)^{2}-0.25\left(\frac{\varepsilon}{d-u-t+\varepsilon}\right)^{8}\right] \phi(u) \mathrm{d} u\right. \\
& +2 \pi R \delta \gamma \int_{d-t}^{d} \phi(u) \mathrm{d} u \\
& \left.+\int_{d}^{\infty} \int_{r_{t}}^{\infty} \frac{8}{3} \frac{\delta \gamma}{\varepsilon}\left[\left(\frac{\varepsilon}{z-t+\varepsilon}\right)^{3}-\left(\frac{\varepsilon}{z-t+\varepsilon}\right)^{9}\right] \cdot 2 \pi r \mathrm{~d} r \phi(u) \mathrm{d} u\right)
\end{aligned}
$$

where $\eta$ is the areal density of asperities, $A_{\mathrm{n}}$ is the nominal contact area, $R$ is the radius of curvature of asperity summits, $\delta \gamma$ is the adhesion energy per unit area for the headdisk interface, $\varepsilon$ is the equilibrium intermolecular spacing, $d$ is the separation of the mean plane of asperity heights, $u$ is the asperity height, $t$ is the thickness of the lubricant layer, $\phi(u)$ is the probability density function of asperity heights. Figure 1 is a schematic diagram illustrating the parameters involved in Eq. 1.

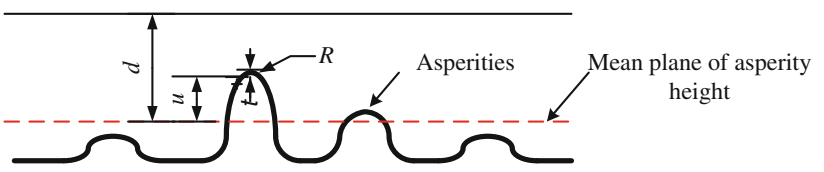

Fig. 1 Schematic diagram of slider and disk surfaces not in contact

In the third integral in Eq. 1, $z$ is the separation of solid surfaces outside the contact region at a radius $r$, and $r_{t}$ is the radius at the intersection of the lubricant and solid, as shown in Fig. 2.

The first integral in Eq. 1 gives the adhesion force arising from non-contacting asperities. It is the integration of the attractive pressure derived from the Lennard-Jones potential over a sphere-shaped profile [12]. The second integral is the contribution of the lubricant-contacting asperities to the adhesion force. The third integral represents the adhesion force due to the solid-contacting asperities. We only consider elastic contact here. For the third integral, the separation $z$ of solid surfaces at a radius $r$ is given by:

$z=\frac{1}{\pi R}\left[a\left(r^{2}-a^{2}\right)^{1 / 2}-\left(2 a^{2}-r^{2}\right) \tan ^{-1}\left(\frac{r^{2}}{a^{2}}-1\right)^{1 / 2}\right]$

where $a=(\omega R)^{1 / 2}$ is the radius of the contact region, $\omega=u-d$ is the interference.

A variety of multi-asperity contact models has been developed giving different relationships between the contact force and the separation of two parallel rough surfaces. Chen and Bogy [13] have shown that the widely used GW (Greenwood-Williamson) model, CEB (Chang-EtsionBogy) model, and KE (Kogut-Etsion) model give little difference provided that the plasticity index of the contact interface is small and only a few of the contacting asperities are fully plastically deformed. Here, we applied the GW model which gives the contact force $P$ as [14]:

$P=\frac{4}{3} \eta A_{\mathrm{n}} E R^{1 / 2} \int_{d}^{\infty}(u-d)^{3 / 2} \phi(u) \mathrm{d} u$

where $E$ is the combined elastic modulus of the two contacting surfaces. Note that by adopting the sub-boundary lubrication model, the contact force is not affected by the lubricant thickness $t$.

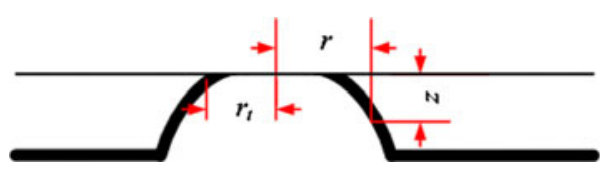

Fig. 2 Schematic diagram of a solid-contacting asperity 
Several assumptions are associated with the subboundary lubrication model: the lubricant is uniformly distributed on the disk roughness and is mobile. The thickness of the lubricant layer is constant since only a small portion of lubricant is displaced under contact with the protruded slider. We also restrict the asperity deformation to be elastic. No bulk deformation and asperity interactions occur. Since only a small protruded part of the slider comes into contact, we also neglect the pressure loss in the air bearing in contact.

\section{Static Simulation of a TFC Slider}

The CMLair static simulation program is used to perform the FH stability investigation. This program uses a QuasiNewton iteration method to search for an equilibrium flying-state of a slider. The Fukui-Kaneko slip correction is adopted in this program to modify the generalized Reynolds equation to account for the rarefaction effects in the air bearing. Patankar's control volume method is applied to discretize the modified Reynolds equation. The equation is then solved using an alternating direction line sweeping method [15]. In the implementation of the sub-boundary lubrication model, each grid in the air bearing surface is regarded as a flat and smooth surface. Combined roughness parameters are applied on the disk. The flying-height is defined as the distance between the air bearing surface and the mean plane of asperity heights on the disk. The asperity height distribution function $\phi(u)$ is assumed to be a normal distribution with a mean of zero and a standard deviation $\sigma$. The integrated interfacial force and torques (including adhesion, contact, and friction forces) are balanced by the air bearing force and the suspension load for an equilibrium solution.

The effects of TFC protrusion are taken into account by modifying the profile of the ABS. For simplicity, we adopt the protrusion profile in Fig. 3 as a base profile and modify this profile proportionally to achieve a higher or lower protrusion profile. The base profile is obtained using the CML TFC program which solves the equilibrium flying attitude and deformation of a TFC slider through an iterative approach [16]. Table 1 gives the roughness and protrusion geometry parameters involved in the simulations.

\section{Results and Discussion}

\subsection{Existence of an Instability Region}

Figure 4 shows the equilibrium minimum flying-height $d^{*}$ as a function of the peak thermal protrusion height for lubricant thickness $t=1.0 \mathrm{~nm}$. Each data point in Fig. 4

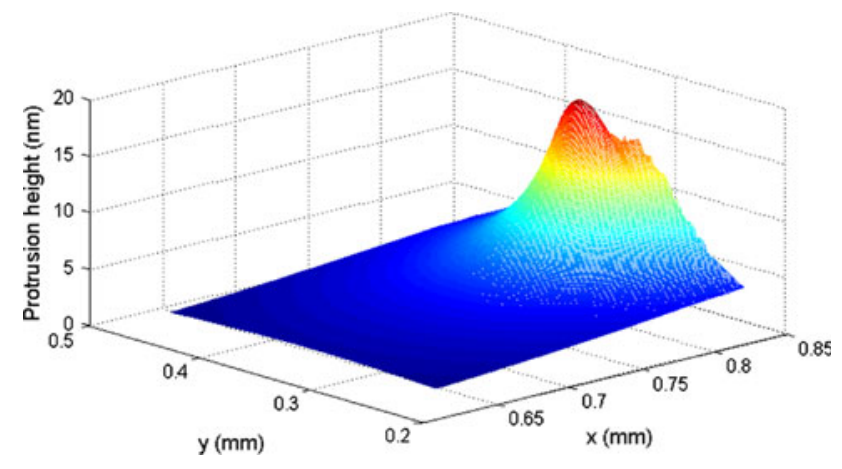

Fig. 3 Thermal protrusion profile obtained through an iterative approach

Table 1 Simulation parameters [8, 13]

\begin{tabular}{ll}
\hline Mean radius of asperities $R(\mu \mathrm{m})$ & 6.384 \\
Asperity density $\eta\left(1 / \mathrm{m}^{2}\right)$ & $9.871 \times 10^{12}$ \\
Standard deviation of asperity heights $\sigma(\mathrm{nm})$ & 0.654 \\
Equilibrium intermolecular distance $\varepsilon(\mathrm{nm})$ & 0.3 \\
Combined elastic modulus $(\mathrm{GPa})$ & 111.59 \\
Friction coefficient & 0.2 \\
Adhesion energy per unit area $\delta \gamma(\mathrm{N} / \mathrm{m})$ & 0.151 \\
Protrusion area $(\mathrm{mm})$ & $0.21 \times 0.24$ \\
Peak thermal protrusion height for the base profile TP* & 17.2927 \\
$\quad(\mathrm{~nm})$ & \\
Lubricant thickness $t(\mathrm{~nm})$ & $0.8,1.0,1.2$ \\
\hline
\end{tabular}

represents an equilibrium flying-state obtained by the CMLair static solver, where a dot means a stable equilibrium state, and a star designates an unstable state. To determine the stability of an equilibrium state, the approach discussed in [7] is used in this study. Briefly, a stiffness matrix of the system, which is defined by the following $3 \times 3$ matrix:

$\left[\begin{array}{ccc}\partial F / \partial z & \partial F / \partial \theta & \partial F / \partial \varphi \\ \partial T_{\theta} / \partial z & \partial T_{\theta} / \partial \theta & \partial T_{\theta} / \partial \varphi \\ \partial T_{\varphi} / \partial z & \partial T_{\varphi} / \partial \theta & \partial T_{\varphi} / \partial \varphi\end{array}\right]$

is evaluated at each equilibrium point. In the stiffness matrix representation, $z$ is the flying-height, $\theta$ is the pitch angle, $\varphi$ is the roll angle, $F$ is the total force in the flyingheight direction, $T_{\theta}$ is the total pitch torque, and $T_{\varphi}$ is the total roll torque. If all three eigenvalues of the stiffness matrix are positive, the system is in a stable equilibrium state. Otherwise, the equilibrium state is regarded as unstable. By "unstable" we mean that the slider cannot stay at its equilibrium state under disturbances and this can result in the slider's unstable motions, such as vibrations. As seen in Fig. 4, the slider's minimum flying-height, denoted as $d^{*}$, is reduced with increasing thermal protrusion. The slider enters into an instability region when 


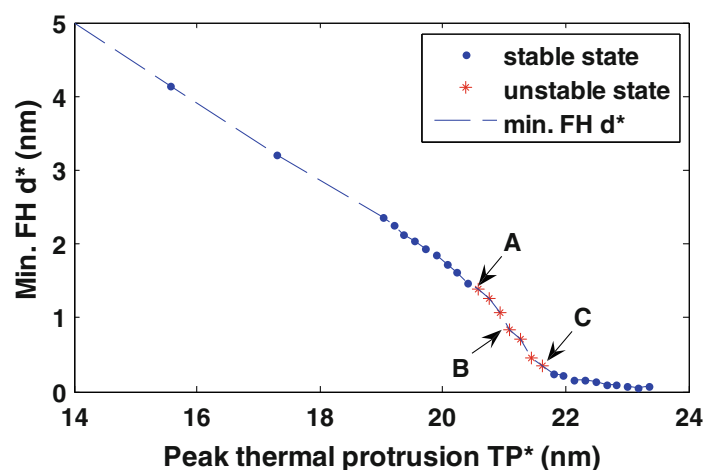

Fig. 4 Equilibrium minimum $\mathrm{FH} d^{*}$ as a function of the peak thermal protrusion $\mathrm{TP}^{*}$. Unstable states are marked with stars

thermal protrusion is beyond a critical point denoted as A. Such an instability region extends from $d^{*}=1.39$ to $d^{*}=0.35$. We denote the end of the instability region as C.

Figure 5 shows the variation of total interfacial force and adhesion force with increasing thermal protrusion. Here, the total interfacial force is defined as the sum of the adhesion force $\left(F_{\mathrm{s}}\right)$ and contact force $(P)$. The unstable points are marked with stars on the adhesion force curve. In the instability region, a rapid increase in the magnitude of the adhesion force with increased protrusion is seen. Since the adhesion force is opposed to the air bearing lifting force, instability occurs when the increase in the air bearing lift cannot balance the growth in adhesion force. In addition, the curve for total interfacial force is almost overlapped with the adhesion force curve at smaller thermal protrusions which shows the contact force is negligible initially. Toward the end of the instability region, the interfacial force curve branches from the adhesion force, showing the growth of contact force at this stage. This can also be seen from Fig. 6, which shows the contact force as a function of $\mathrm{TP}^{*}$. As the contact force adds up to the lifting force of the air bearing, this fast increase in the

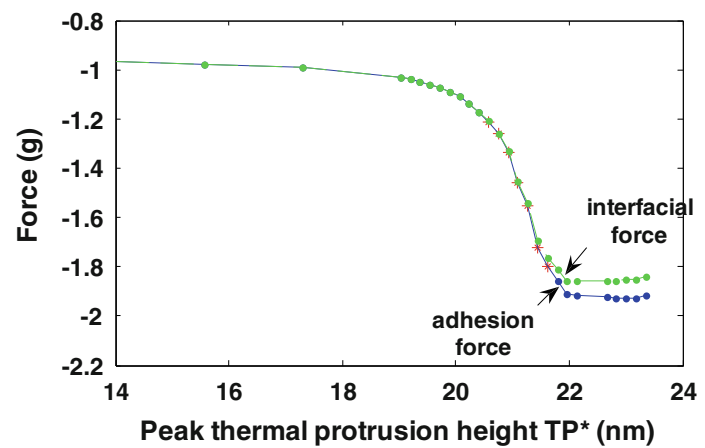

Fig. 5 Interfacial force and adhesion force at equilibrium for different thermal protrusions. Unstable states are marked with stars

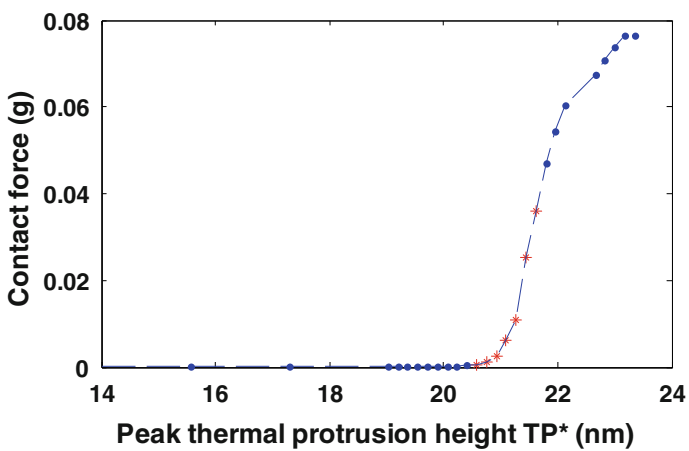

Fig. 6 Contact force at equilibrium for different thermal protrusions. Unstable points are marked with stars

contact force at the end of the instability region contributes to balancing the adhesion force and helps to restore HDI stability.

Figure 7 shows the adhesion force on a single asperity as a function of the normalized flying-height $h(h=\mathrm{d} / \sigma)$. The three contributions to the adhesion force (i.e., the three integrals in Eq. 1) are also plotted. The magnitude of the adhesion force is first growing with an increasing rate as we reduce the flying-height, and then reaches a maximum. The increasing rate is maintained at the maximum as we further reduce the flying-height, and then decreased. Here, we mark the minimum flying-height $d^{*}$ at points $\mathrm{A}$ and $\mathrm{C}$ from Fig. 4 by the two vertical dashed lines. $h_{\mathrm{A}}$ is located at the segment of the curve where the magnitude of adhesion force is growing with the greatest rate. At this stage, the HDI is most endangered due to the adhesive effect. $h_{\mathrm{C}}$ is located at the segment of the curve where the increasing rate in the adhesion force magnitude is starting to decrease. This indicates the restoration of HDI stability at point $\mathrm{C}$, on the one hand, is due to the growth in contact

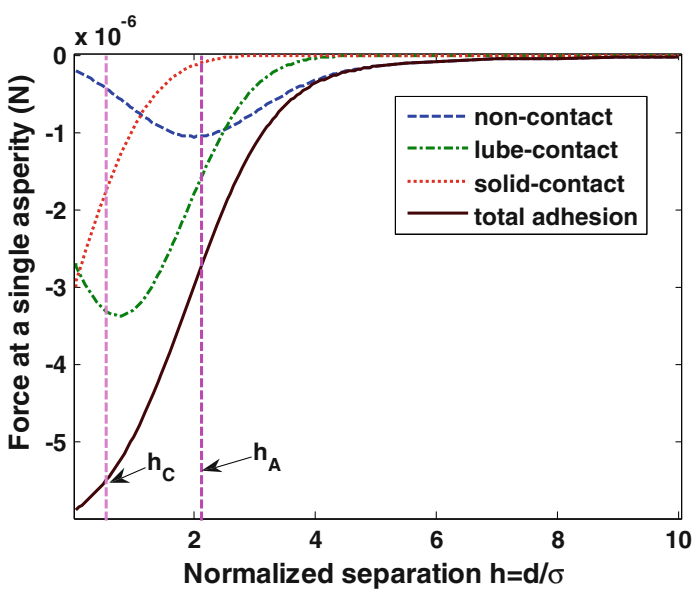

Fig. 7 Adhesion force at a single asperity for different flying-heights The minimum flying-height for states $\mathrm{A}$ and $\mathrm{C}$ are marked with $h_{\mathrm{A}}$ and $h_{\mathrm{C}}$ 


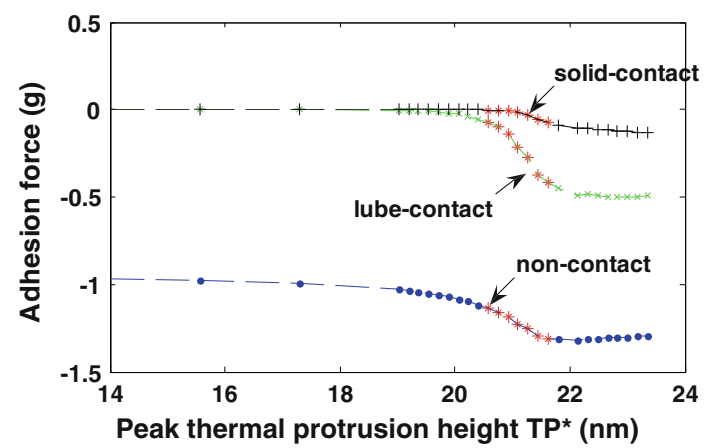

Fig. 8 Components of the adhesion force for different thermal protrusions. Unstable points are marked with stars

force as shown in Fig. 6, and on the other hand, is also attributed to this reduced increasing rate in adhesion force indicated by Fig. 7 .

In Fig. 8, we take a closer look at the increase in the magnitude of adhesion force near the instability region. We plot the contributions from non-contacting, lubricant-contacting, and solid-contacting asperities on the same scale. It is obvious that the adhesion force at lubricant-contacting asperities accounts for the largest part of the increase in adhesion force at the instability region.

\subsection{Effect of Lubricant Thickness}

Since we have found that the fast growth in the adhesion force is associated with the HDI instability found in simulations, different lubricant thicknesses may affect the range of the instability region as $t$ is related to the adhesion force according to Eq. 1. Here, we investigate three lubricant thicknesses $t=0.8,1.0$, and $1.2 \mathrm{~nm}$ with the same roughness parameters and protrusion profile. The relationships between the minimum flying-height $d^{*}$ and the peak thermal protrusion $\mathrm{TP}^{*}$ for the three cases are shown in Fig. 9, where

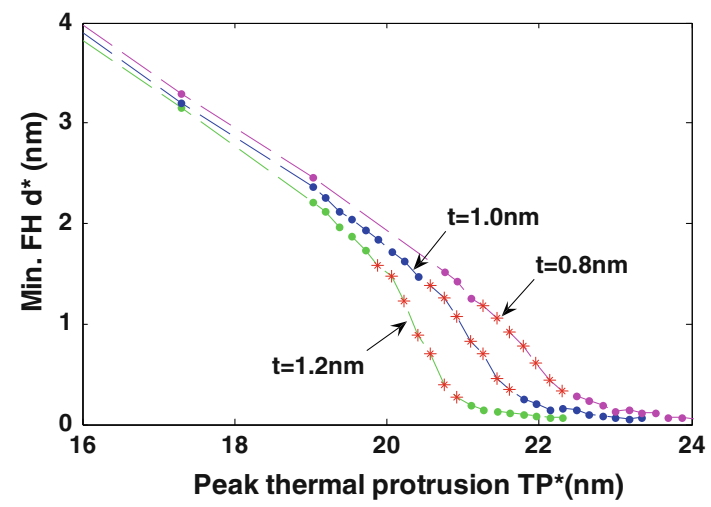

Fig. 9 Minimum flying-height $d^{*}$ at equilibrium at different thermal protrusions for $t=0.8,1.0$, and $1.2 \mathrm{~nm}$ the unstable states are marked with stars. As shown in Fig. 9, at the same peak thermal protrusion (the ABS is exactly the same), $d^{*}$ drops with increasing lubricant thickness. Also, the critical $\mathrm{TP}^{*}\left(\mathrm{TP}^{*}\right.$ at the starting point of the instability region) is $21.27,20.58$, and $19.89 \mathrm{~nm}$ for $t=0.8,1.0$, and $1.2 \mathrm{~nm}$, respectively. Therefore with a thinner lubricant layer, we are allowed to actuate the slider to a higher protrusion while maintaining a stable HDI. The corresponding critical $d^{*}\left(d^{*}\right.$ at the starting point of the instability region) for $t=0.8 \mathrm{~nm}$ is $1.18 \mathrm{~nm}$, the lowest among the three, which means that in this case the slider can get closest to the disk, without losing the HDI stability. This result also shows that although it seems that in a lube-surfing recording scheme, we may even relax the limit on lubricant thickness to some extent [4], as the slider is allowed to make contact with the lubricant, a thicker lubricant can result in a less stable HDI: the slider may lose stability at a relatively higher flyingheight.

\subsection{Effect of Protrusion Profile}

The profile of thermal protrusion is another factor that can be associated with the instability due to adhesion forces. Consider the three protrusion profiles B, I, and J shown in Fig. 10, which are viewed from the trailing edge of the slider and pass through the point with the peak protrusion height. Curve B is calculated using the CML TFC program and taken as a base profile. By increasing or decreasing the base area of case B, we get a steepened profile, e.g., curve I, or a flattened profile, such as curve J. Note that B, I, and J have the same peak protrusion height. Suppose the slider is fixed at zero pitch and roll, and moves toward the disk, we are expecting that the steepest profile results in a relatively small rate of change in the adhesion force with decreasing flying-height, since a smaller part of the protruded area is in closer contact with the disk.

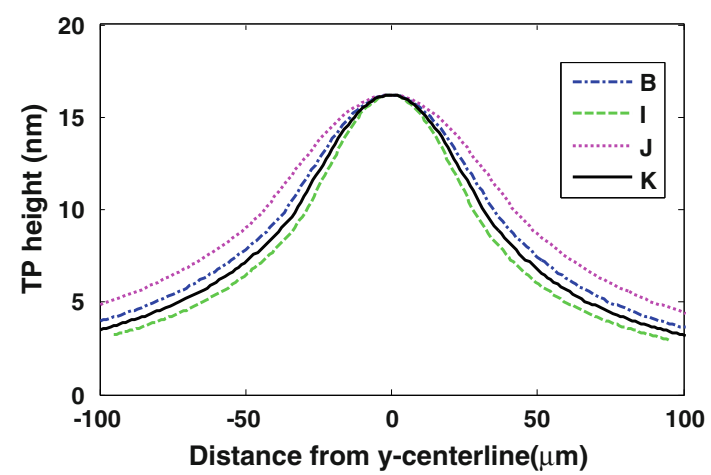

Fig. 10 Protrusion profiles of cases B, I, J, K with different base areas 


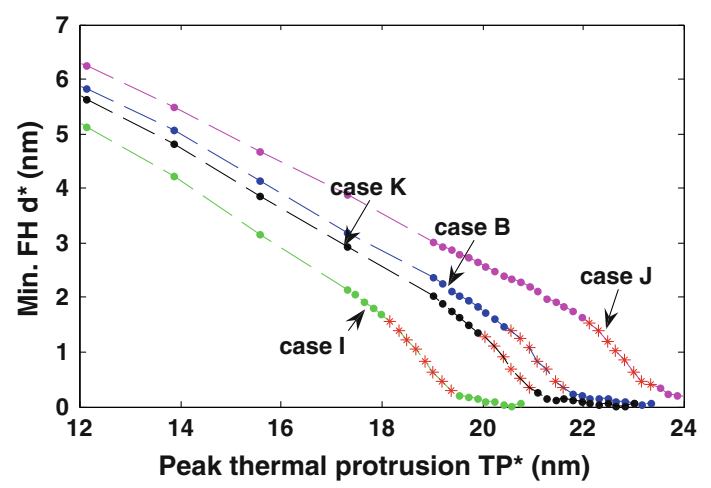

Fig. 11 Minimum flying-height $d^{*}$ at equilibrium for different thermal protrusions for cases B, I, J, and $\mathrm{K}$

Here, we take $t=1.0 \mathrm{~nm}$ and calculate the instability regions for cases $\mathrm{B}$, I, and $\mathrm{J}$, respectively. The thermal actuation for each case is modified by increasing the protrusion height proportionally with respect to the original profiles shown in Fig. 10. Interestingly, the result is not monotonic with respect to the steepness of the protrusion profiles, as shown in Fig. 11. The critical $d^{*}$ marking the onset of instability in case I, instead of being lower due to the steepened protrusion, is $1.55 \mathrm{~nm}$, i.e., higher compared with $1.39 \mathrm{~nm}$ of case B. For a specific TP*, which means we are keeping peak protrusion height the same for both the cases, case I gives a lower flying-height, even when the interfacial forces are negligible (according to Fig. 7, the adhesion force takes effect when $d$ is less than $5 \mathrm{~nm}$ ). This shows that for the same TP*, the air bearing is less stiff in case I (the stiffness of the air bearing is defined as the change in the air bearing force per unit flying-height change). Therefore, the increase in the air bearing lift in case I fails to balance the fast growth in adhesion force due to a less stiff air bearing, and this results in a higher critical $d^{*}$.

In comparison, case $\mathrm{J}$, which has the flattened profile, enters the instability region at $d^{*}=1.53 \mathrm{~nm}$, almost the same as case I. As shown in Fig. 11, case J has a stiffer air bearing for the same TP*. Thus, the stiffer air bearing in case $\mathrm{J}$ compensates the faster increase in the adhesion force, and keeps the critical $d^{*}$ almost unchanged compared with case I. Suppose the base area for case $B$ is $A_{B}$, by increasing the base area from $0.8 \mathrm{~A}_{\mathrm{B}}$ (case I) to $1.2 \mathrm{~A}_{\mathrm{B}}$ (case $\mathrm{J}$ ) with a step size of $0.05 \mathrm{~A}_{\mathrm{B}}$, we found the profile of case $\mathrm{K}$ with a base area of $0.9 \mathrm{~A}_{\mathrm{B}}$ (as shown in Fig. 10), has a minimum critical $d^{*}$ of $1.27 \mathrm{~nm}$, as shown in Fig. 11.

\section{Conclusion}

By including the sub-boundary lubrication model, we find an instability region exists as a TFC slider comes into contact with the disk lubricant or the disk surface roughness through increased thermal protrusions: the slider maintains a stable equilibrium state in light contact with the lubricant; but stability is lost when the contact gets more severe; and the stability returns as the slider enters deep-contact with the disk roughness. Existence of such an instability region is related to a fast growth of adhesion force which is not balanced by the increase in the air bearing lifting force. This growth in the adhesion force is mainly caused by the increase in the lubricant-contacting adhesion force.

The effects of lubricant thickness and protrusion steepness on this instability region are investigated. A thinner lubricant layer is beneficial for reducing the critical flyingheight beyond which instability occurs. As for the protrusion steepness, although less sensitivity to the increased adhesion force is expected for a steeper protrusion, this may trade off with a less stiff air bearing. Thus, optimization in TFC design is necessary for achieving the largest possible stability region in the lubricant-contact recording scheme.

Open Access This article is distributed under the terms of the Creative Commons Attribution Noncommercial License which permits any noncommercial use, distribution, and reproduction in any medium, provided the original author(s) and source are credited.

\section{References}

1. Juang, J.Y., Nakamura, T., Knigge, B., Luo, Y., Hsiao, W.C., Kuroki, K., Huang, F.Y., Baumgart, P.: Numerical and experimental analyses of nanometer-scale flying height control of magnetic head with heating element. IEEE Trans. Magn. 44(11), 3679-3682 (2008)

2. Juang, J.Y., Chen, D., Bogy, D.B.: Alternate air bearing slider design areal density of $1 \mathrm{~Tb} / \mathrm{in}^{2}$. IEEE Trans. Magn. 42(2), 241246 (2006)

3. Aoki, K., Watanabe, T.: Nonlinearity of thermal spacing control in hard disk drives. IEEE Trans. Magn. 45(2), 816-821 (2009)

4. Liu, B., Zhang, M.S., Yu, S.K., Hua, W., Ma, Y.S., Zhou, W.D., Gonzaga, L., Man, Y.J.: Lubricant-surfing recording and its feasibility exploration. IEEE Trans. Magn. 45(2), 899-904 (2009)

5. Thornton, B.H., Bogy, D.B.: A parametric study of head-disk interface instability due to intermolecular forces. IEEE Trans. Magn. 40(1), 337-344 (2004)

6. Suh, A.Y., Polycarpou, A.A.: Dynamic adhesive instability of sub-five nanometer head-disk interfaces. In: Proceedings of the 13th Mediterranean Conference on Control and Automation, pp. 139-146, 2005

7. Gupta, V., Bogy, D.B.: Dynamics of sub-5 $\mathrm{nm}$ air bearing sliders in the presence of electrostatic and intermolecular forces at the head disk interface. IEEE Trans. Magn. 41(2), 610-615 (2005)

8. Li, N., Zheng, L., Bogy, D.B., Meng, Y.: Flyability and durability test of dynamic fly-height sliders at 1-nm. Tribol. Trans. 53(2), 212-218 (2010)

9. Vangipuram Canchi, S., Bogy, D.B.: Slider dynamics in the lubricant-contact regime. IEEE Trans. Magn. 46(3), 764-769 (2010) 
10. Hua, W., Liu, B., Yu, S., Zhou, W.: Nanoscale roughness contact in a slider-disk interface. Nanotechnology 20, 285710 (2009)

11. Stanley, H.M., Etsion, I., Bogy, D.B.: Adhesion of contacting rough surfaces in the presence of sub-boundary lubrication. Trans. ASME 112, 98-104 (1990)

12. Muller, V.M., Derjaguin, B.V., Toporov, Yu.P.: On two methods of calculation of the force of sticking of an elastic sphere to a rigid plane. Colloids Surf. 7, 251-259 (1983)

13. Chen, D., Bogy, D.B.: Intermolecular force and surface roughness models for air bearing simulations for sub-5 nm flying height sliders. Microsyst. Technol. 13, 1211-1217 (2007)
14. Greenwood, J.A., Williamson, J.B.P.: Contact of nominally flat surfaces. Proc. Roy. Soc. Lond. A 112(1442), 300-319 (1966)

15. Lu, S.: Numerical simulation of slider air bearings. Doctoral Dissertation (1997)

16. Zheng, J., Bogy, D.B., Zhang, S., Yan, W.: Effects of altitude on the thermal flying height actuation. In: Proceedings of the ASME/ STLE International Joint Tribology Conference, 2009 